\title{
MÁRGENES DE ACCIÓN: EL PROCESO ARTESANAL COMO MÉTODO DE PROYECTO EN LA ARQUITECTURA CONTEMPORÁNEA DE PARAGUAY
}

LIMITSOFACTION: THEARTISAN PROCESS AS PROJECTMETHODINTHE CONTEMPORARY ARCHITECTURE OF PARAGUAY

José Luis Uribe Ortiz

RESUMEN Durante los últimos veinte años la contingencia política y las crisis económicas han orientado la mirada de la escena arquitectónica contemporánea hacia contextos periféricos, promoviendo una arquitectura surgida bajo ámbitos de escasez y articulada por su abundante cultura material. Lo anterior ha definido una nueva narrativa arquitectónica orientada por la incidencia del proceso artesanal como parte activa de la obra arquitectónica. Una arquitectura al margen la cual se articula a partir de la reaparición del quehacer material del artesano y el saber ancestral del hombre como una condición contemporánea de la arquitectura. Es ahí donde el presente artículo se inserta, a partir de una serie de planteamientos que orientan los márgenes de acción de la nueva arquitectura contemporánea en Paraguay, cuya narrativa arquitectónica será desplegada a partir de la lectura de tres casos de estudio agrupados en los materiales característicos proporcionados por ese territorio.

PALABRAS CLAVES artesanal; Paraguay; escasez; materia; hapticidad

SUMMARY During the past twenty years, political contingency and frequent economic crises within Paraguay have oriented their view of contemporary architecture towards peripheral contexts, resulting in a style of architecture which emerged under scarce resources, but articulated by its abundant material culture. The above has defined a new architectural narrative, oriented by the artisanal process as an active part of architectural work. Paraguay enjoys an "architecture on the margin," which is typified by the reappearance of material craftsman paired with the ancestral knowledge of man as a contemporary condition. This is the context of the present article, based on a series of approaches that guide the margins of action of the new contemporary architecture in Paraguay, whose architectural narrative will be illustrated by the analysis of three case studies, grouped in the characteristic materials provided by that territory.

KEY WORDS artisan; Paraguay; shortage; matter; hapticity 
PROYECTO, PROGRESO, ARQUITECTURA

17

\section{DEL AISLAMIENTO COMO CONDICIÓN}

A nivel sudamericano, Paraguay se inserta en medio del continente caracterizándose por no tener contacto con el mar y estar rodeado por las respectivas culturas de tres países: Bolivia al norte, Brasil al este y Argentina al sureste, sur y suroeste. Esta particular ubicación le otorga a su cultura un carácter introspectivo y una autonomía que busca distanciarse respecto al imaginario e identidad de los países que conforman sus márgenes. Este encapsulamiento se ha manifestado en distintos episodios de su historia, como la ínsula socialista del Doctor Francia durante su época de gobernación nacional, la actitud bélica que los ha llevado a combatir contra los países vecinos ${ }^{1}$ o el enclaustramiento político de Stroessner durante la dictadura más extensa de Latinoamérica².

En términos demográficos, Paraguay es uno de los países con menor cantidad de habitantes comparado con sus pares latinoamericanos, presentando una irregular distribución en su territorio la cual se encuentra concentrada en la región oriental del Paraguay, dando cuenta de la condición inhóspita de la región occidental caracterizada por su cálida temperatura y densa selva Según Britez y Numan en términos económicos, Paraguay ha llegado a poseer el Producto Interno Bruto más bajo de Latinoamérica, Transparencia Internacional lo ha considerado el país más corrupto del continente y el segundo a nivel mundial. También ha llegado a ser el uno de los países más pobres de Latinoamérica ${ }^{3}$.

Considerando estos antecedentes, a escala Latinoamérica, Paraguay manifiesta una condición menguada al compararse con el resto de los países latinoamericanos en aspectos geográficos, políticos, demográficos y económicos respecto al resto de los países. El aislamiento y encierro geográfico que condiciona al país repercute en sus distintas vertientes disciplinares, derivando en una constante exclusión de Paraguay.

A escala de país, su estructura interna manifiesta una fragmentación y polarización en las distintas disciplinas de las ciencias sociales, lo que deriva en la desigualdad como un tema que ha afectado a los habitantes de Paraguay durante las últimas décadas y que se manifiesta a través de la corrupción, escasez y pobreza. 
1. Artesano trabajando en la fabricación de ladrillos compactados en tierra cruda para la Vivienda Takurú de José Cubilla. Piribebuy. Paraguay. 2015.

2. Proceso de obra Facultad de Arquitectura, Diseño y Arte de la Universidad Nacional de Asunción diseñado por el Gabinete de Arquitectura. San Lorenzo. Paraguay. 2017.

A pesar de desplegar esta serie de antecedentes poco alentadores y como un contrapunto a ellos, durante las últimas décadas Paraguay ha logrado un desarrollo cultural basado en lo propio y alejado de referencias externas, condición que ha incidido en la particular manera de hacer arquitectura.

\section{DE LA CULTURA MATERIAL Y LOS PROCESOS ARTESANALES}

La condición aislada que manifiesta Paraguay sumado a su escasez de recursos se ve contratado por su generosa cultura material, lo cual ha sido destacado por diversos autores ${ }^{4}$. Para el presente estudio es pertinente aproximarse al concepto de imaginación material desplegado por Gastón Bachelard en su libro "El agua y los sueños. Ensayo sobre la imaginación de la materia" ${ }^{5}$ ya que permite inferir la apertura que entrega la materia en un proceso creativo. En arquitectura, la materia puede informar al proyecto, así como puede definir la expresión arquitectónica de la obra. La información que entrega la materia al proceso de proyecto se puede establecer en dos líneas: Por un lado, mediante la exploración material guiada por el arquitecto y por otro lado, mediante la intuición, destreza y proximidad con la materia que entrega un artesano (figura 1).

Consideraremos el protagonismo de la figura del artesano en la narrativa arquitectónica contemporánea del Paraguay y es lo que definirá el hilo conductor del presente estudio. Tomaremos distancia de la imagen de los arquitectos que han recibido una formación artesanal ${ }^{6} \mathrm{y}$ que incorporaron ese conocimiento a sus procesos de diseño, ya que esta interpretación ha desdibujado la autoría entre arquitecto y artesano. Sumaremos lo planteado por

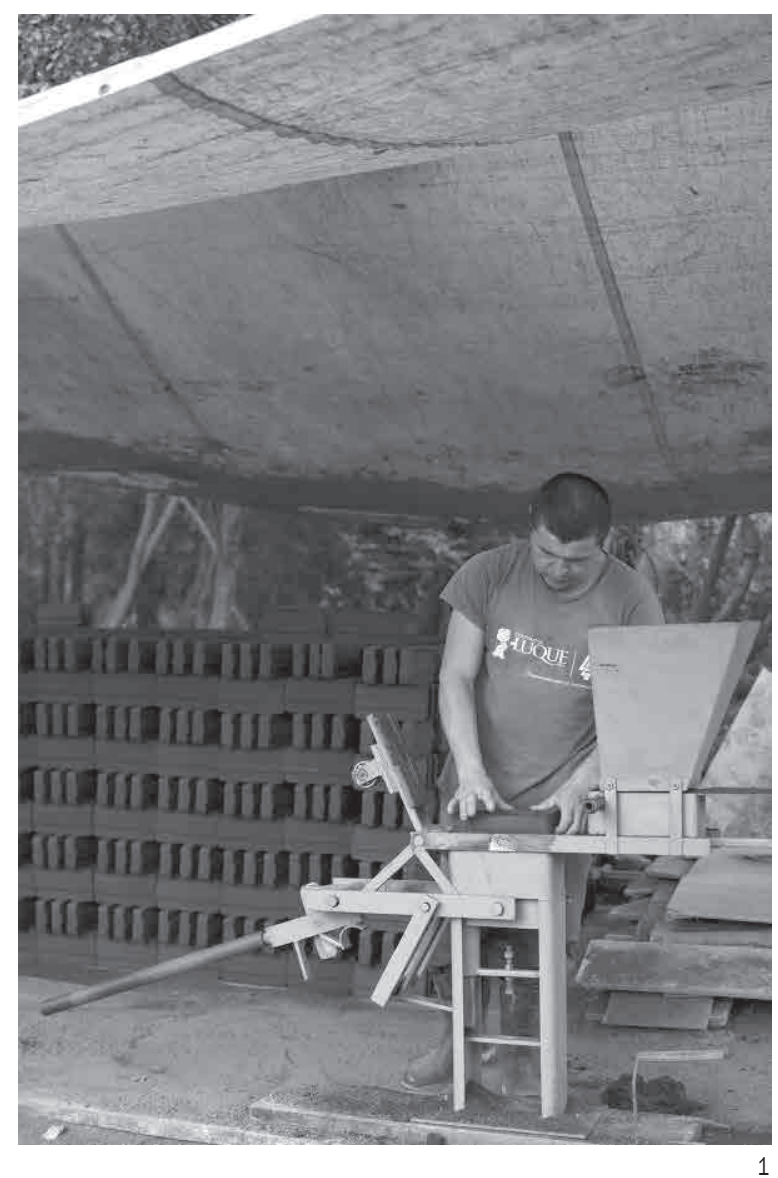

Juhani Pallasmaa respecto a que en un trabajo creativo, tanto el artista como el artesano se involucran directamente con sus cuerpos y experiencias existenciales, más

4. Juan Giuria, pone en valor sus maderas, piedras y ladrillo de Paraguay planteando que "Se calcula que más de un setenta por ciento de su superficie está cubierta por bosques de magnificas maderas, aptas para toda clase de obras. En cuanto a los materiales de origen pétreo es indiscutible que existen canteras de excelente piedra arenisca como lo comprueban ciertas obras llevadas a cabo por los jesuitas, en las que aún pueden verse enormes paños de muros ejecutados con bloques de aquella piedra. Durante la época hispana, y aun en años posteriores, se hizo gran consumo de arcilla, ya en forma de adobe 0 simplemente como tierra apisonada. El empleo del ladrillo cocido se desarrolló a partir de la independencia". Para el investigador Ramón Gutiérrez, el contexto geográfico influenció en una cultura material basada en el tanteo, acumulación y atesoramiento de materiales, destacando que "Ios materiales disponibles desde un comienzo en el territorio paraguayo fueron los de recolección: Madera, caña, paja, barro y piedra".

5. BACHELARD, Gastón. El agua y los sueños. Ensayo sobre la imaginación de la materia. Madrid: Editorial Fondo de Cultura Económica de España. 2005.

6. Me refiero a arquitectos como Carlo Scarpa que se formó durante veinte años con el adiestramiento artesanal de los maestros vidrieros de Murano o el caso de Peter Zumthor, quien reconoce sus orígenes como el hijo de un artesano (ebanista en Basel) que se formó como carpintero. 


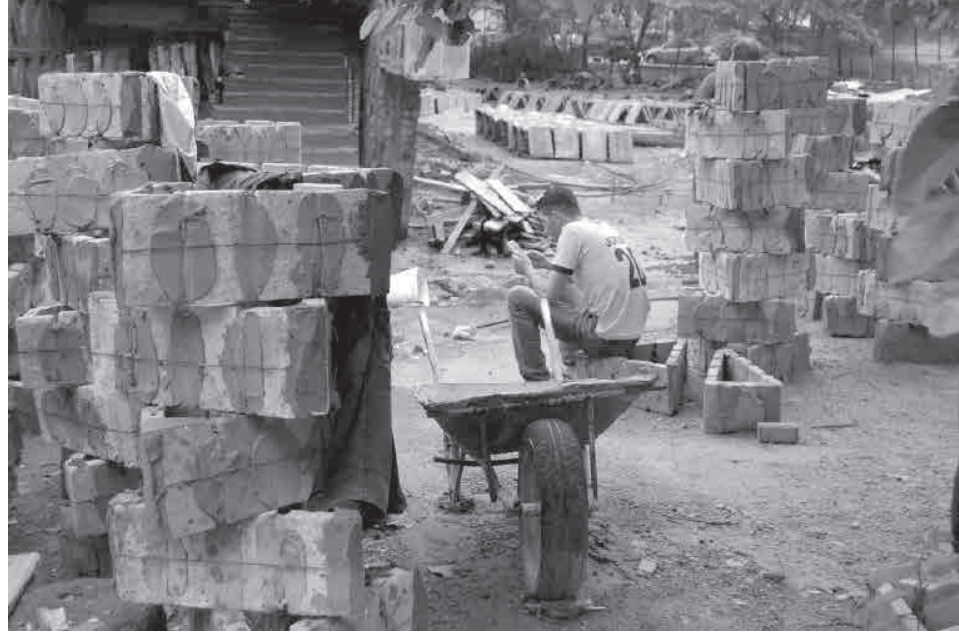

2 acción del cuerpo sobre la materia, práctica adiestrada a través del tiempo ${ }^{9}$. Esta postura podemos asociarla a la particular manera de hacer arquitectura en Paraguay, donde a lo largo de su historia se ha caracterizado por la búsqueda de un lenguaje arquitectónico local a partir de la utilización de los materiales que el entorno entregaba.

Según Gutiérrez durante la arquitectura paraguaya en el periodo de dominación hispánica tres grandes sectores asumen la tarea de realizar la arquitectura: Los maestros de obras, los arquitectos de órdenes religiosas y los arquitectos de ingenieros militares. Centrándonos en los maestros de obras, Gutiérrez pone en valor el hecho de que "están especialmente capacitados a través del proceso de aprendiz-oficial-maestro de los gremios españoles, quienes en Paraguay encontraron posibilidades de desarrollar sus aptitudes con nuevos materiales y sistemas constructivos"10. Esta relación aprendiz - oficial - maestro se basa en la idea de lo artesanal planteada por Sennett, donde la finalidad es establecer un aprendizaje intelectual basado en la práctica adiestrada en el tiempo y en las constantes repeticiones, a lo que se suma una gran capacidad de adaptabilidad de los artesanos paraguayos a las condiciones económicas, tecnológicas y materiales de la época11 (figura 2).

Los procesos artesanales que forman parte de la expresión arquitectónica en el Paraguay empiezan a obedecer a la lógica material que entrega el entorno inmediato del lugar, la incidencia del saber artesanal como parte de una sinergia basada en el intercambio de experiencias y experimentación desarrollada entre el artesano constructor y el arquitecto. Se define así una mayor relación de la obra con el emplazamiento y la realidad humana que lleva a cabo esa arquitectura, distanciándose de la lógica de una arquitectura solo basada en la recolección

7. PALLASMAA, Juhani. Los ojos de la piel: La arquitectura y los sentidos. Barcelona: Editorial Gustavo Gili. 2012.

8. Esta idea refleja la unión de la mano y la cabeza aplicada sobre la materia, lo que permite que se produzca un vínculo entre la práctica manual y la capacidad para innovar y resolver problemas en la actualidad a partir de una lógica material.

9. SENNETT, Richard. El artesano. Barcelona: Editorial Anagrama. 2009.

10. GUTIÉRREZ, Ramón. Historia de la arquitectura del Paraguay 1537-1911. Asunción: Editorial Municipalidad de Asunción. 2010, p.51.

11. Cecilia Román y Andrés Careaga plantean que "Los arquitectos paraguayos que han aportado a la disciplina primero tuvieron que ingeniárselas para enseñar nuevas maneras de hacer a sus albañiles. (...) Empieza un proceso de re elaboración, entendiendo primeramente la técnica tradicional, dominándola para luego transformarla y sobre todo, para poder explicarla y ponerla en práctica satisfactoriamente". 


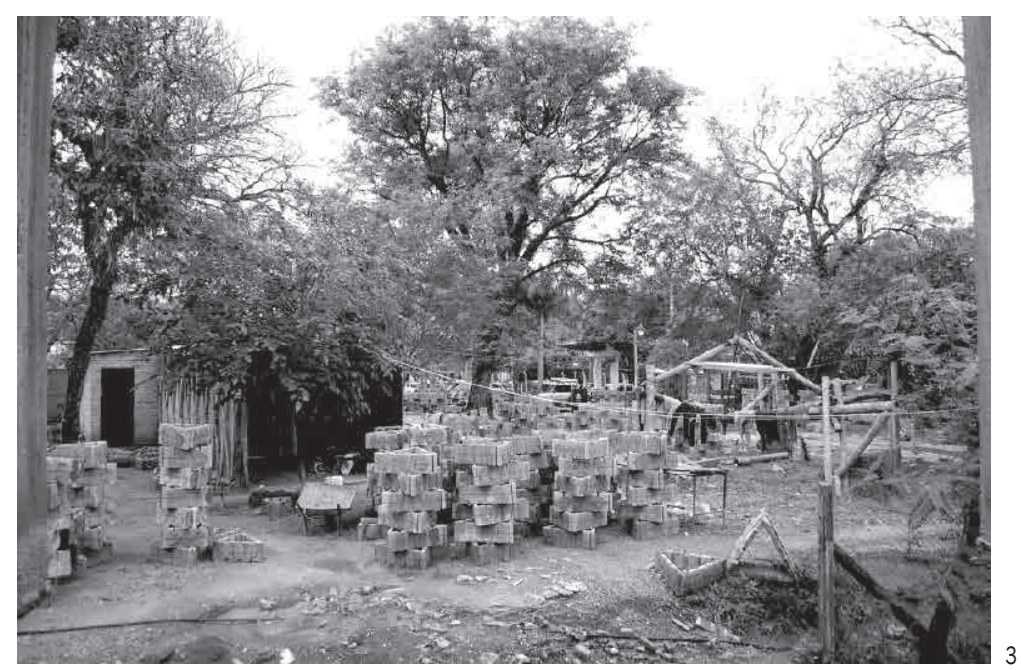

3. Prefabricados y modulación en el proceso de obra Facultad de Arquitectura, Diseño y Arte de la Universidad Nacional de Asunción diseñado por el Gabinete de Arquitectura. San Lorenzo. Paraguay. 2017. 4. Clínica Médica y Vivienda de Estudio Elgué. Asunción. Paraguay. 2015.

5. Boceto detalle constructivos Clínica Médica y Vivienda del Estudio Elgué. Asunción. Paraguay. 2015 material que caracterizó sus inicios. Para la editora Florencia Rodríguez la manifestación contemporánea de la arquitectura contemporánea en Paraguay "no es una arquitectura del detalle dibujado, sino que reclama del ensuciarse los pies en el barro y buscar un alto grado de sofisticación de recursos matéricos"12. Es así como esta manera de proyectar basada en la manipulación material donde de una manera deslavada, cruda, bruta y alejada del ordenador es posible aproximarse a las expresiones plásticas que caracterizan la arquitectura contemporánea en el Paraguay (figura 3).

\section{LA ARQUITECTURA CONTEMPORÁNEA EN PARAGUAY} Atendiendo los libros sobre historia y teoría arquitectónica latinoamericana o las exposiciones y bienales asociadas hacia la disciplina, la arquitectura de Paraguay siempre se ha mantenido relegada o simplemente estaba ausente, insistiendo con esta condición aislada mencionada al inicio del estudio. No era valorada dentro de la producción arquitectónica del contexto latinoamericano y no se reconocía una manera propia de hacer arquitectura. A finales de los años noventa, posteriormente a la dictadura política de Stroessner y bajo una seguidilla de corruptos gobiernos de transi- ción, surge una particular manera de hacer arquitectura que se distanciaba a la producción desarrollada en Latinoamérica. De la mano de los arquitectos Javier Corvalán y Solano Benítez irrumpen una serie de pequeñas obras de arquitectura que aportan un lenguaje propio basado en la cultura material originaria del Paraguay, el reconocimiento de nuevas maneras de interpretar materiales corrientes y aplicarlos en obras otorgando una dignidad espacial, plástica y material, procesos en los cuales el arquitecto desarrollaba un trabajo colaborativo con la experiencia propia de los artesanos.

Lo anterior ha generado una masa crítica que ha sostenido el interés internacional por la escena arquitectónica contemporánea en Paraguay ${ }^{13}$, lo que es parte de un lento proceso de transmisión de conocimientos que tuvo que pasar por diversas etapas para posteriormente posicionarse no solo en el ámbito nacional sino que también en lo internacional. Es así como esta manifestación arquitectónica inserta en el corazón de Sudamérica contiene características de lo que Frampton reconoce como un Regionalismo Critico $^{14}$ y que Zaera Polo lo agrupa bajo el concepto de Material Fundamentalists en la gráfica del artículo Ya Bien Entrado el Siglo XXI ¿Las Arquitecturas del

12. RODRÍGUEZ, Florencia. Nuevas prácticas en Asunción. En: PLOT. Buenos Aires: Piedra, Papel y Tijera SA, número 26, 2015, p. 28.

13. Íbidem, p. 26. Según Rodríguez, está apertura arquitectónica en Paraguay "tiene un correlato con ciertos intercambios que se fueron generando en el tiempo a través de talleres, conferencias, publicaciones y conversaciones. Podríamos destacar algunos hechos como la selección de finalistas que se realizó en ocasión del segundo premio Mies van der Rohe para Latinoamérica. Angelo Bucci de Brasil, Rafael lglesia de Argentina, Alejandro Aravena de Chile y Solano Benítez de Paraguay se consolidaron desde aquel momento como un grupo representativo de algunas de las transformaciones y propuestas de la región. Este punto abrió el dialogo entre las personas y entre el cuerpo de obras que obtuvo visibilidad y nuevas capas de significación cuando se activó su puesta en relación".

14. Como reacción al estilo internacional, Frampton postula el regionalismo crítico acercándonos a una arquitectura que contrarresta la carencia de lugar y falta de identidad en la arquitectura moderna mediante el uso de contexto geográfico y material del edificio como partida de proyecto, retomando la labor del artesano que desde sus procesos manuales define las particularidades de una arquitectura que lucha con la tendencia a uniformar. 


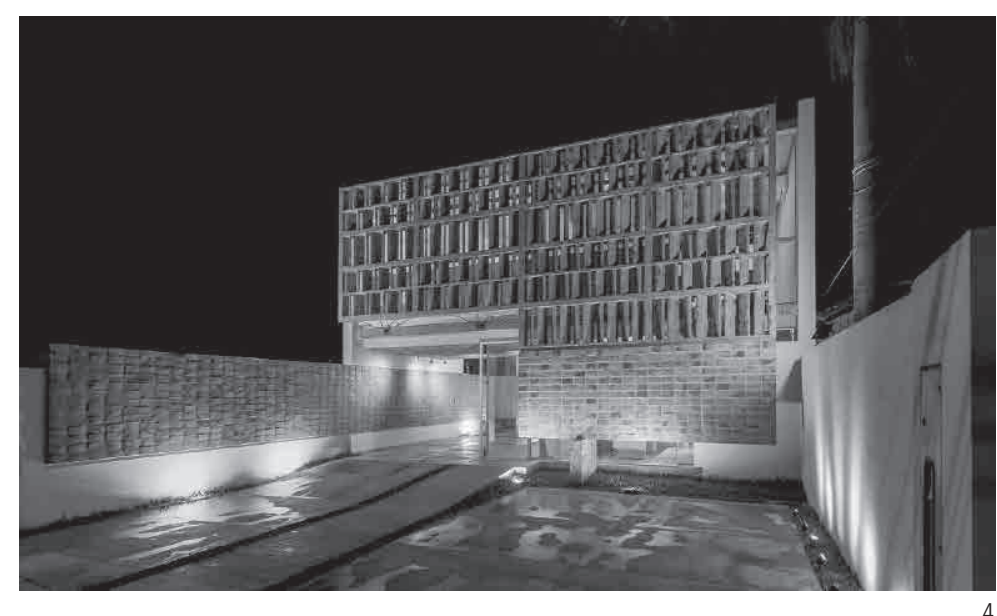

Post-Capitalismo? ${ }^{15}$. En ese sentido, el presente artículo no pretende realzar la etiqueta de arquitectura social o de arquitectura de reciclaje que durante las últimas décadas ha recibido el trabajo de algunos de los despachos. Por contra, se estructurará el análisis arquitectónico a modo de un relato que busca acercarse a la complejidad de los procesos de proyecto estudiados y al impacto en la expresión de su arquitectura, de lo artesanal ligado a la obra, de un peso y papel fundamentales

\section{Lectura de casos}

La presente lectura de casos se enmarca en el trabajo de tres obras de despachos paraguayos de los cuales interesan determinadas parcialidades de las obras. Los casos revisados tienen como elemento en común la proyectación del objeto arquitectónico en contextos de abundancia material y escasez económica, sumado a la incorporación de artesanos que tienen participación directa del proyecto arquitectónico. Esta participación artesanal por lo general se establece junto al arquitecto en un dialogo basado en el intercambio de experiencias en torno a los procedimientos manuales que intervienen en la arquitectura, los que vienen de la mano de alfareros, albañiles, canteros y carpinteros, quienes desarrollan su quehacer a partir de la materia que los rodea. A continuación realizaremos una lectura de elementos arquitectónicos singulares de cada obra, lo que se desglosa mediante tres materiales característicos en ese país, orientando la lectura a partir de la incidencia del proceso artesanal en el método arquitectónico.
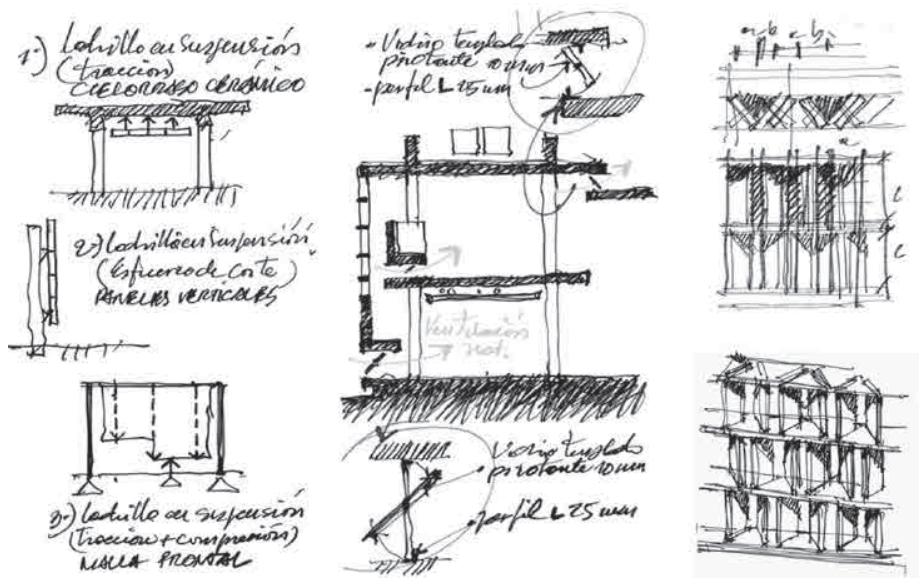

5

\section{Del ladrillo como tejido}

Iniciaremos la lectura considerando el ladrillo como madera y nos centraremos en el parasol de la Clínica Médica y Vivienda proyectada por el Estudio Elgué plantea un tejido cerámico en la fachada oeste apoyada en un solo punto de equilibrio, vinculando la situación espacial de la calle, la construcción nueva y la pre existente. Este elemento arquitectónico define una pantalla permeable cuya función contempla, por un lado, atender el exceso de luz y calor proveniente del oeste y, por otro lado, configurar un elemento que define la continuidad de la fachada del conjunto, el cual también amortigua la relación entre el espacio público y el espacio privado (figura 4).

Este tramado monomaterial se estructura a partir de una geometría rectangular que se sobrepone al edificio, definiendo una fachada traslucida la cual está confeccionada a partir de un elemento pesado como es el ladrillo ${ }^{16}$, cuyo lenguaje material se manifiesta a partir de dos tipos de grano: el primero, correspondiente a un muro hermético de ladrillo apoyado sobre un machón, suspendiendo en el aire el peso de toda la fachada. Sobre esa geometría cerrada se reconoce el segundo grano del ladrillo, el cual se adhiere mediante una trama de paneles de ladrillos ubicados de manera vertical y zigzagueante, dejando un espacio para la ventilación entre cada ladrillo. La membrana cerámica queda suspendida y no toca ni los muros del edificio preexistente ni el suelo natural donde se emplaza el objeto arquitectónico. El acceso al edificio define un vacío que permite el dialogo de la arquitectura con la escala del habitante y está conformado a partir de un recorte rectangular en el extremo inferior izquierdo de la geometría mayor (figura 5).

15. ZAERA POLO, Alejandro. Ya Bien Entrado el Siglo XXI ¿Las Arquitecturas del Post-Capitalismo. En: El Croquis. Madrid: Editorial El Croquis, número 187, 2016, p 252-287.

16. Hay que considerar que en esta obra se utiliza el ladrillo de descarte como partido material. Corresponde al ladrillo que al posarse sobre el nivel del suelo natural de los patios de secado se contrae por efecto del calor, generando una deformación de la unidad material. Al ser un ladrillo defectuoso tiene un costo menor, promoviendo una arquitectura basada en la economía de recursos. 
N18_ARQUITECTURAS AL MARGEN

22
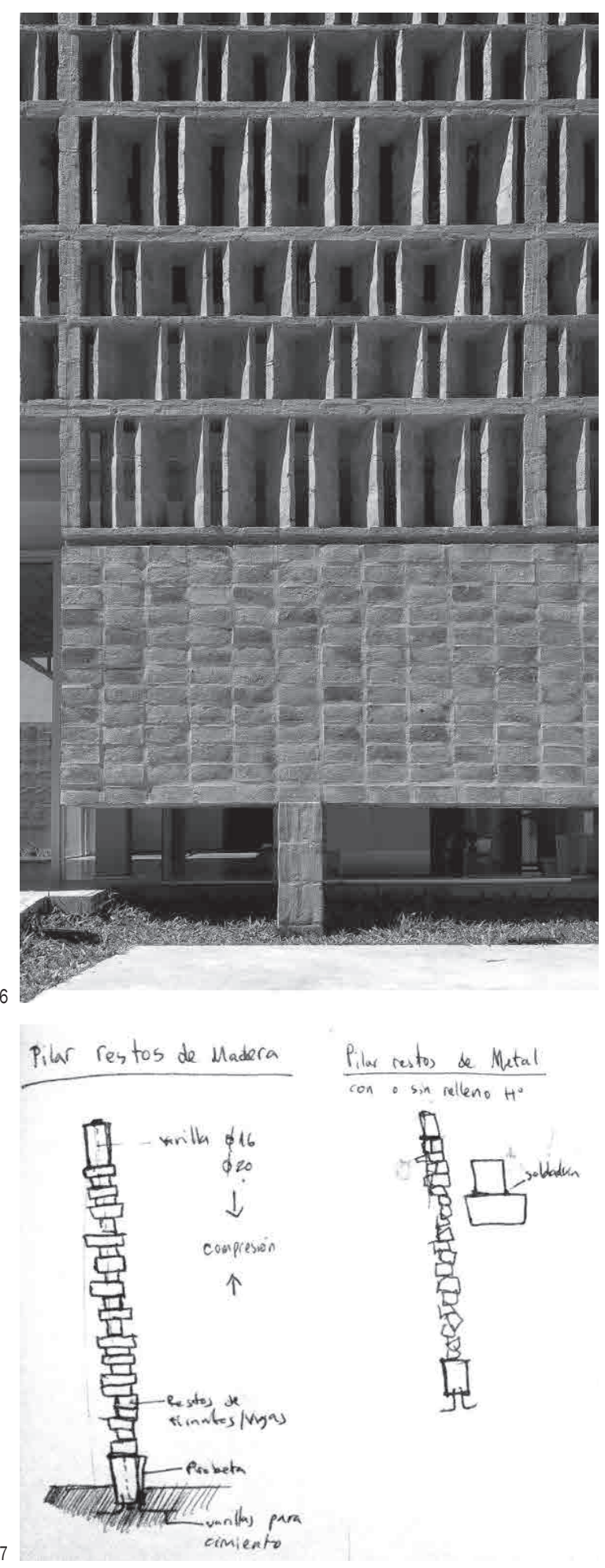

6. Clínica Médica y Vivienda del Estudio Elgué. Asunción. Paraguay. 2015.

7. Boceto detalle constructivo Casa - Taller Las Mercedes de Lukas Fuster. Asunción. Paraguay. 2012.

8. Sección Casa - Taller Las Mercedes de Lukas Fuster. Asunción. Paraguay. 2012.

9. Sección Casa - Taller Las Mercedes de Lukas Fuster. Asunción. Paraguay. 2012.

10. Boceto detalle constructivo Vivienda Takurú de José Cubilla. Piribebuy. Paraguay. 2015.
Cabe destacar el hecho de que la expresión plástica del edificio viene definido por un proceso de ensayo y error desarrollado en terreno, direccionado a partir de una serie de pruebas materiales y posteriores decisiones de proyecto en las cuales participaron el arquitecto y el artesano, otorgándole a esos ladrillos de descarte una mayor dignidad, aproximándonos a la idea del defecto como expresión arquitectónica. Otro punto destacable es la geometría del elemento arquitectónico que se asocia a la geometría de las cesterías indígenas de Paraguay, caracterizadas por su construcción basada en el tejido y el entramado (figura 6).

\section{De la madera como apilamiento}

La lectura del segundo caso considera la madera como materia aplicada a un elemento arquitectónico estructural como es el pilar, desarrollado a partir de un lenguaje material local asociado a un quehacer artesanal. Nos centraremos en el pilar de la casa estudio Las Mercedes de Lukas Fuster quien proyecta un elemento arquitectónico particular como consecuencia de una intervención que toma como partido la acción de resta material desarrollada sobre una antigua vivienda ubicada en el casco antiguo de Asunción, donde la materia reciclada vuelve a insertarse en la vivienda a partir de una serie de intervenciones de escala doméstica, las cuales reconocen la lógica de que la materia no se crea ni se destruye, solo se transforma ${ }^{17}$. El único pilar inserto en la vivienda se plantea bajo un sistema constructivo que apila y enhebra trozos de madera de descarte a compresión mediante una vara de acero pretensado dando forma al fuste del pilar. Tanto la basa como el capitel están definidos por dos probetas de hormigón ubicadas a la llegada del pilar al suelo y cielo de la vivienda, otorgándole al pilar una dignidad propia de una columna que soporta parte de la vivienda, tal cual lo hace el horcón como elemento estructural presente en la vivienda rural guaraní (figura 7).

Materialmente esta pieza está compuesta mediante una sumatoria de trozos correspondientes a los restos más pequeños de los tirantes de madera que se han

17. DE LAVOISIER, Antonine-Laurent. Tratado de Química (Reedición). Valladolid: Editorial Maxtor. 2009. 


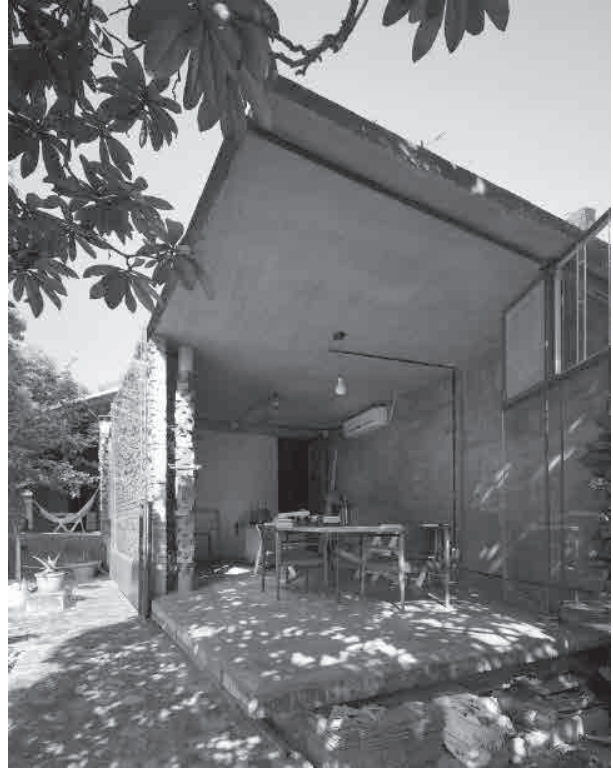

8

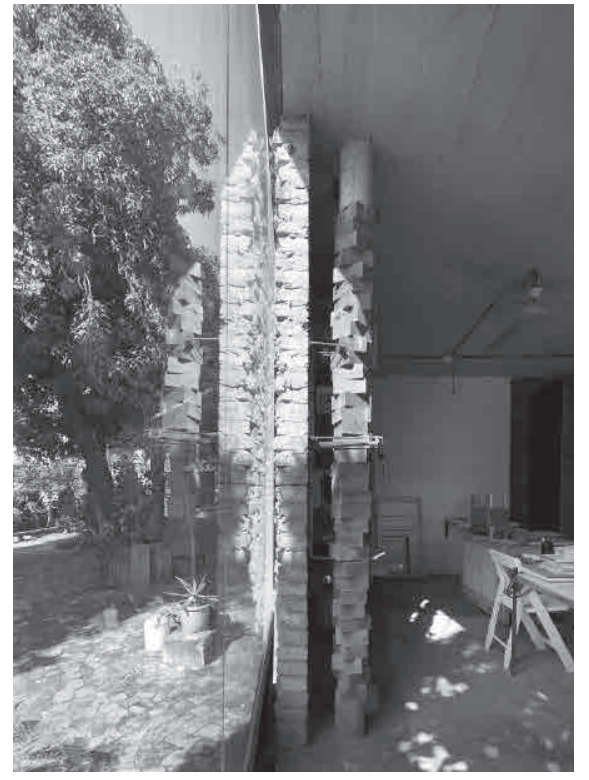

9

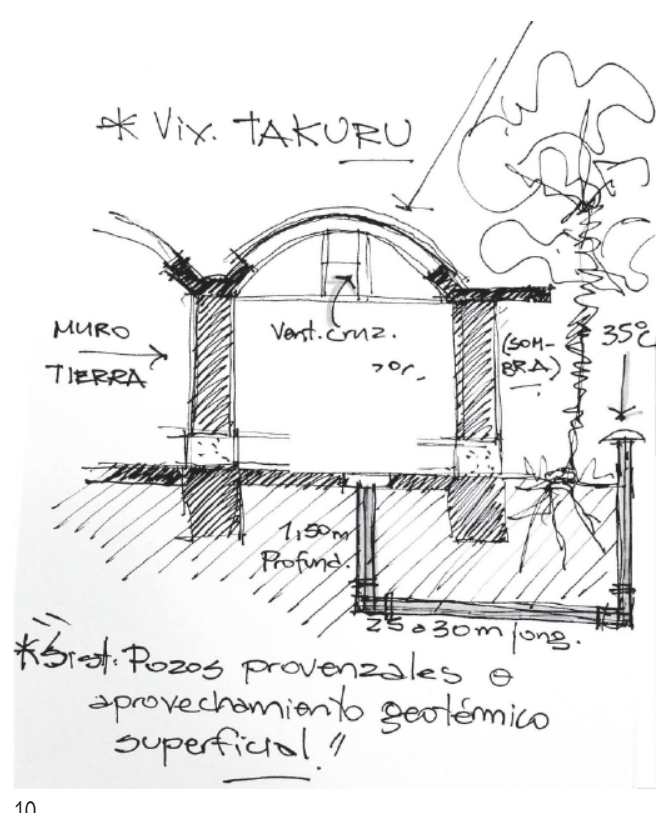

10 reutilizado y que se concentran en un solo elemento arquitectónico atendiendo a la acción centrifuga del arquitecto, similar a la actitud planteada por Ramón Gutiérrez respecto al valor, tanteo y acumulaciones de elementos materiales presente en los indígenas paraguayos. La composición material del pilar replantea el lenguaje plástico de la columna, el cual por lo general tiene una composición monomaterial continua. En este caso está constituido a partir de la superposición de piezas, definiendo un elemento que media entre la losa y el cielo, similar al apilamiento material de la apacheta, acumulación de piedras que los indios y mestizos de algunas zonas andinas. Finalmente, el elemento arquitectónico desarrollado por Fuster es un pilar a partir de la acción del apilar, juego de palabras que insiste en la acumulación y superposición que define la actitud arquitectónica (figura 8).

El Pilar de la casa estudio Las Mercedes es parte de una obra arquitectónica de carácter procesual, que se construye con el tiempo, atendiendo la constante incidencia del clima en el emplazamiento así como la mirada atenta de su habitante al entorno cotidiano y los elementos funcionales que componen su arquitectura. La casa se entiende como un laboratorio en el que cada intervención arquitectónica atiende a una serie de pruebas materiales y estructurales que inciden en su habitar doméstico. En este caso la figura del arquitecto y habitante de la obra se transforma en una suerte de Animal laborans en palabras de Sennett, en cuyo entorno doméstico es posible divisar diversos trabajos manuales que dan cuenta de la afinidad de Fuster por la artesanía y los oficios, donde su casa estudio es un constante ensayo de formas de construir (figura 9).

\section{De la tierra como compactación}

Para cerrar la lectura de casos nos referiremos a la tierra como materia de proyecto a partir del muro exterior de la Vivienda Takurú de José Cubilla, que corresponde a un elemento arquitectónico conformado mediante la compactación de tierra y que construye el perímetro que define el total de la vivienda. Este elemento se basa en un sistema constructivo local como es el tapial, antigua técnica que consiste en la construcción de muros portantes a partir de tierra arcillosa húmeda compactada a golpes mediante un pisón, la cual define su base gracias a un encofrado de madera. La excavación de una gran fosa aporta la materia prima para construir gran la extensión del muro y el suelo se convierte en paramento. La materia adquiere geometría para trazar la vivienda a partir de un muro que entrega continuidad y ritmo a una secuencia espacial que descansa en su vacío central (figura 10).

El muro exterior como parte de la caja muraria de esta obra, define una condición hermética de la cotidianeidad del interior de la vivienda, en contraste con la apertura de la extensión del paisaje natural sobre la cual se emplaza. Esta idea de un elemento perimetral conformado a partir de un material compactado permite establecer una relación con la alfarería indígena paraguaya, en específico con las vasijas, tanto en la concepción de un vacío 


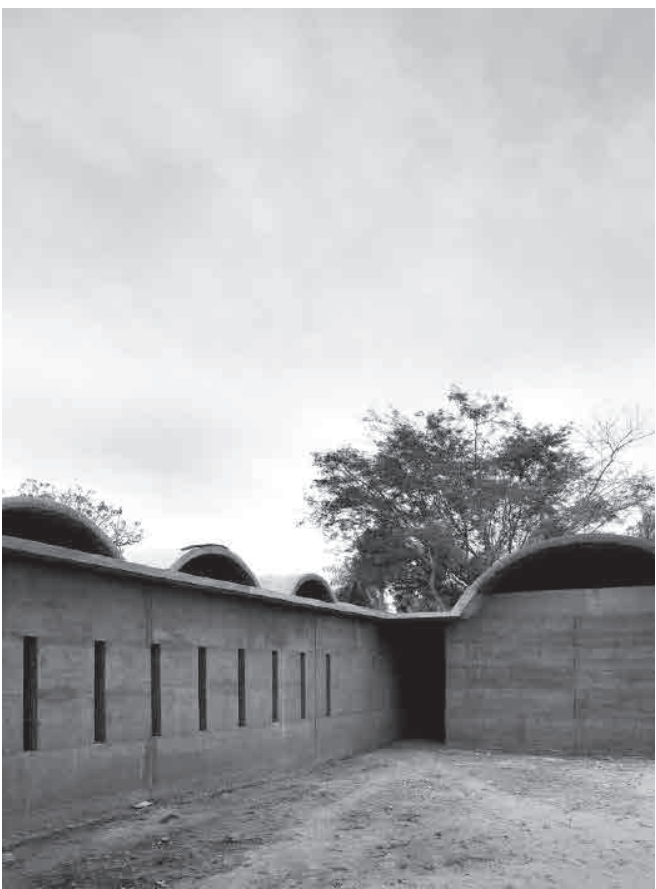

11
11. Vivienda Takurú de José Cubilla. Piribebuy. Paraguay. 2015.

12. Vivienda Takurú de José Cubilla. Piribebuy. Paraguay. 2015.

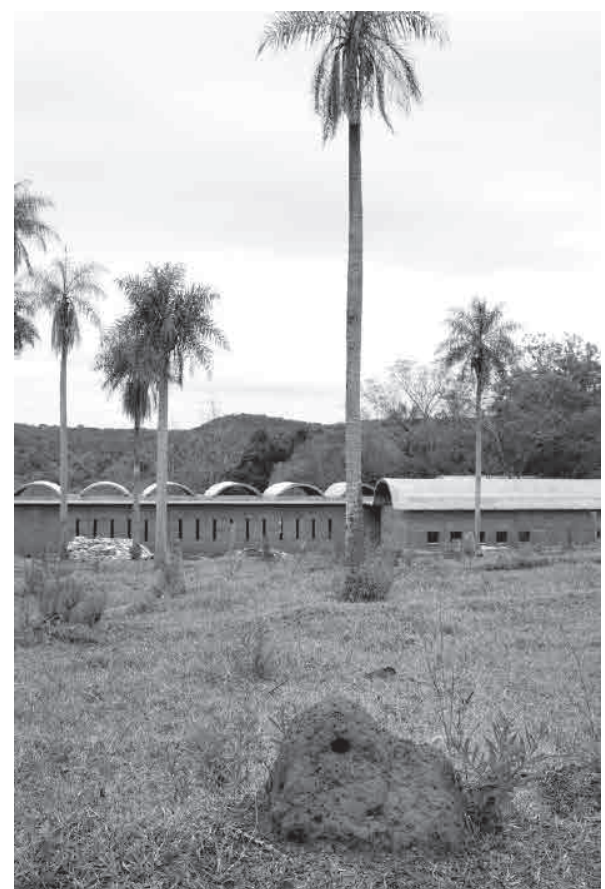

12 central que constituye el muro de tapial como definición arquitectónica, así como en el vacío interior presente en la composición de las vasijas de cerámica indígena. En ambos casos se reconoce una intención por definir un elemento contenedor y un espacio contenido, en los cuales se busca conformar un aire, un posible interior, como en el caso de la vivienda Takurú donde un pequeño patio interior se equipara con el caso de la vasija y el espacio vacío dentro de un contenedor material (figura 11).

Otra aproximación entre el muro de tapial y la cerámica indígena producida en ese territorio tiene que ver con la condición material de ambos casos, donde la terracota rojiza y las estrías de las juntas de los muros son similares a las incisiones propias de la decoración de la alfarería guaraní. A partir del tapial como sistema constructivo es posible observar en el muro los estratos materiales, su granulometría y un sugerente color rojizo, elementos que median entre la arquitectura y las bondades del suelo donde se emplaza la obra. Durante el proceso de construcción de este elemento arquitectónico es posible atender como se desarrolla de manera contemporánea una antigua práctica artesanal de construcción donde confluye el cuerpo, la materia el lugar y la arquitectura. En el proceso de manufacturado de las vasijas indígenas se asoma una condición escultural propia del hacer con las manos. La vasija es un contenedor que contiene aire y los muros de la Vivienda Takurú trazan un contenedor que también contiene aire, pero es un aire que espera ser habitado.

Materialmente es un elemento arquitectónico constituido a partir del peso, apropiándose de la lógica constructiva basada en la estereotomía del basamento de Semper mediante un elemento monomaterial. Un muro confeccionado a partir del constante golpeteo en el proceso de obra en terreno, proceso manual desarrollado entre el arquitecto junto al artesano, donde detona la expresión material primitiva de la obra, que se manifiesta de manera cruda y bruta hacia la intemperie (figura 12).

Este elemento arquitectónico corrobora la actitud proyectual de los arquitectos contemporáneos en Paraguay de construir con los elementos recogidos desde un alrededor planteado por Gutiérrez y la lógica de lo centrípeto a partir de la definición de un elemento arquitectónico concentrado y denso, constituido por la riqueza material del lugar.

\section{ALGUNAS NOTAS A MODO DE CONCLUSIONES}

De la incidencia del cuerpo en la expresión arquitectónica Posterior a esta revisión de casos es necesario atender la importancia que Peter Zumthor otorga al cuerpo en 
la arquitectura. El efecto sensorial que la materia detona en el arquitecto le permite "reunir cosas y materiales del mundo para que, unidos, creen este espacio. Para mí se trata de algo así como una anatomía. En realidad al hablar del cuerpo lo hago en el sentido literal de la palabra"18. Las definiciones de Zumthor incorporan una componente material y una participación del cuerpo en la definición formal, insinuando una interacción manual en el proceso constructivo. Es así como por un lado, la componente material entrega una información que es decodificada por el cuerpo del habitante al recorrer la obra ${ }^{19}$, lo que Pallasmaa reconoce como las experiencias sensoriales pasan a integrarse a través del cuerpo $^{20}$. Por otro lado, Alvar Aalto otorga importancia a lo humano como parte del proceso arquitectónico donde "el factor humano siempre ha sido parte de la arquitectura y que, en un sentido más profundo, ha sido incluso indispensable, pues sin su contribución no se podría expresar la riqueza de la vida y sus cualidades positivas"21.

Según lo que plantea tanto Zumthor, Pallasmaa y Aalto podríamos inferir que el artesano desde su corporalidad en contacto con la materia aporta a la expresión arquitectónica de la obra construida, lo que en el caso de la arquitectura contemporánea en Paraguay tiene una incidencia directa en su lenguaje arquitectónico, la cual se gesta a partir de la experimentación material en terreno, tal como describe Rodríguez "esta no es una arquitectura del detalle dibujado, sino que reclama del ensuciarse los pies en el barro y buscar un alto grado de sofisticación de recursos matéricos"22. Es el cuerpo el que define la escala habitable de los espacios durante la etapa de proyectación, la expresión plástica del edificio en su proceso de construcción y la definición material del objeto arquitectónico una vez construido.
De la hapticidad en la arquitectura

Finalmente, la participación del artesano dentro de la materialización del proyecto aporta una cualidad táctil a la experiencia de recorrer la secuencia espacial de una arquitectura. Para Le Corbusier, la piedra, el ladrillo, la madera o el hierro tienen potenciales distintos, energías sentimentales y físicas, donde el contacto permanente del habitante con la materia es una necesidad fundamental ${ }^{23}$ Pallasmaa reconoce dos variables que articulan la interacción sensitiva del habitante con el objeto arquitectónico: en primer lugar el tacto como elemento sensorial que integra nuestra experiencia del mundo con nuestro interior. En segundo lugar, las superficies de los materiales naturales que nos permiten convencernos de la veracidad de la materia, en una época caracterizada por la tersura de la construcción estándar. La superficie de la materia define los diversos espacios del objeto arquitectónico y orienta una secuencia espacial basada en experiencia de habitar en contacto material con la obra. Deplazes pone en valor la descripción del arquitecto Mandfred Sack respecto a la dimensión háptica de la arquitectura: "una y otra vez se hace presente la sensualidad de lo material, de la materia: cómo se siente al tacto, a la vista. Si es mate o brillante, como huele, si es dura, blanda, elástica, fría o caliente, lisa o rugosa, cuáles son sus colores, que estructuras exhibe en su superficie"24.

Lo anterior puede entenderse como la sinceridad de la superficie material, la cual siempre se muestra de una manera directa, cruda y visceral hacia el habitante, cuya definición táctil viene como consecuencia de un trabajo artesanal en el proceso de construcción de la obra arquitectónica, tal como el arquitecto y artista paraguayo Carlos Colombino trabaja las superficie de sus matrices para desarrollar su xilografías, cuyas texturas "surgen a cada golpe de la cuchilla. Los planos se multiplican.

18. ZUMTHOR, Peter. Atmosferas. Barcelona: Editorial Gustavo Gili. 2006, p. 22.

19. PALLASMAA, J. Op. cit., supra, nota 7, p. 68. En palabras de Pallasmaa "La piel lee la textura, el peso, la densidad y la temperatura de la materia".

20. Ibídem, p. 50.

21. AALTO, Alvar. Alvar Aalto: De palabra y por escrito. Madrid: El Croquis Editorial. 2000.

22. RODRÍGUEZ, Florencia. Op.cit., supra, nota 12, p. 28.

23. LE CORBUSIER. Cuando las catedrales eran blancas. Buenos Aires: Editorial Poseidón. 1963.

24. DEPLAZES. Andrea. Construir la arquitectura. Del material en bruto al edificio. Barcelona: Editorial Gustavo Gili. 2010, p. 19.

25. PLÁ, Josefina. Carlos Colombino, pintor paraguayo. En: Humboldt. Hamburgo: Editorial Goethe Institut, número 39, 1969. p. 97. 


\section{N18_ARQUITECTURAS AL MARGEN}

26

El relieve se enriquece y el color concurre para sugerir luces y sombras"25. Un manera de operar similar es la desarrollada por los arquitectos paraguayos contemporáneos, quienes reconocen en los procesos vernaculares de construcción una serie de valores, tal como muestran en este estudio las obras analizadas, donde muros, suelos y cielos están configurados a partir de elementos repetitivos como trozos de ladrillo, madera o piezas a base de tierra comprimida aportando rugosidades y estrías en la expresión de la superficie de la arquitectura, lo que Rodríguez asocia como "una especie de patchwork monocromo que se percibe empatado pero que encierra un catálogo de diferentes texturas. Esto crea atmosferas y experiencias indiscutiblemente vernáculas"26. Una experiencia habitable única, guiada por una gran carga material que ha sido moldeada con suma habilidad por cada artesano cuyo oficio se ha construido con el tiempo, tal cual se ha ido construyendo la identidad de la arquitectura contemporánea en Paraguay.
De la lógica centrípeta de construir con la materia que hay alrededor

Los elementos arquitectónicos revisados dan cuenta de distintas acciones materiales y constructivas desarrolladas a partir de la colaboración entre el arquitecto y los artesanos. Podemos mencionar la acción de entramar en el parasol de la Clínica Médica y Vivienda de Estudio Elgué, el apilar que define el pilar de la Casa Estudio Las Mercedes de Lukas Fuster y el densificar que se puede reconocer en la expresión muraria de la Casa Takurú. Cada una de estas acciones materiales han sido desarrolladas con las manos, conformando una lógica constructiva centrípeta que tiende a concentrar referencias directas e indirectas de la cultura local del Paraguay en estos elementos arquitectónicos. Una actitud distinta a la condición de aislamiento que presenta Paraguay como cultura y que tiende a centrifugar las referencias culturales externas. 
PROYECTO, PROGRESO, ARQUITECTURA

27

\section{Bibliografía citada:}

AALTO, Alvar. Alvar Aalto: De palabra y por escrito. Madrid: El Croquis Editorial. 2000.

BACHELARD, Gastón. El agua y los sueños. Ensayo sobre la imaginación de la materia. Madrid: Editorial Fondo de Cultura Económica de España. 2005. BENITEZ, Solano. Gabinete de arquitectura. En: ARQ Santiago: Ediciones ARQ, número 51, 2002. ISSN: 0716-0852.

BRITEZ, Edwin. NUMAN, Javier. El Paraguay actual. 1998- 2010. Asunción: El Lector. 2010

CORVALÁN, Javier. Un fin del mundo. En: RITA Madrid: Editorial Red Fundamentos, número 1, 2014.

CUBILLA, José. Vivienda Takurú. En: Catalogo XX Bienal Panamericana de Arquitectura de Quito. Quito: Editorial Colegio de Arquitectos de Ecuador. 2016. DEPLAZES. Andrea. Construir la arquitectura. Del material en bruto al edificio. Barcelona: Editorial Gustavo Gili. 2010.

ELGUÉ, Luis. Cuando la historia se reinventa. En: AU - Arquitectura e Urbanismo. Sao Paulo: Editorial Pini, número 247, 2014. ISSN: $0102-8979$.

ELGUÉ, Luis. Sobre ladrillos y ladridos. En: Summa +. Buenos Aires: DONN SA, número 137. 2014.

FUSTER, Lukas. Casa - Taller Las Mercedes. En: PLOT. Buenos Aires: Piedra, Papel y Tijera SA, número 26. 2015.

GIURIA, Juan. La Arquitectura en el Paraguay. Buenos Aires: Instituto de Arte Americano e Investigaciones Estéticas.1950.

GUTIERREZ, Ramón. Historia de la arquitectura del Paraguay 1537-1911. Asunción: Editorial Municipalidad de Asunción. 2010.

LE CORBUSIER. Cuando las catedrales eran blancas. Buenos Aires: Editorial Poseidón. 1963.

PALLASMAA, Juhani. Los ojos de la piel: La arquitectura y los sentidos. Barcelona: Editorial Gustavo Gili. 2012.

PLÁ, Josefina. Carlos Colombino, pintor paraguayo. En: Humboldt. Hamburgo: Goethe Institut, número 39. 1969. ISSN: 0018-7615.

RIOS, Silvio. La vivienda guaraní. Aproximación a la vivienda guaraní en las cuencas de los ríos Paraguay, Paraná y Uruguay. San Lorenzo: FADA UNA Ed. 2016. RODRÍGUEZ, Florencia. Nuevas prácticas en Asunción. En: PLOT. Buenos Aires: Piedra, Papel y Tijera SA, número 26, 2015.

ROMÁN, Cecilia. ARTEAGA, Cesar. Participación paraguaya en exposiciones arquitectónicas internacionales desde 1998 hasta 2016. Asunción: Tesis de final de grado. Facultad de Arquitectura, Arte y Diseño Universidad Nacional de Asunción. 2017.

SARGI0TTI, Ricardo. Casa Gertopán. En: Revista 30-60 Cuadernos de Arquitectura. Córdoba: Editorial I+P, número 18. 2014.

SENNETT, Richard. El artesano. Barcelona: Editorial Anagrama. 2009.

ZAERA POLO, Alejandro. Ya Bien Entrado el Siglo XXI ¿Las Arquitecturas del Post-Capitalismo. En: El Croquis. Madrid: Editorial El Croquis, numero 187. 2016. ZUMTHOR, Peter. Pensar la arquitectura. Barcelona: Editorial Gustavo Gili (Reedición). 2014.

José Luis Uribe Ortiz (Talca, Chile, 1979) Arquitecto por la Escuela de Arquitectura de la Universidad de Talca (Chile. 2007). Master en Teoría y Práctica del Proyecto de Arquitectura por la Escuela Técnica Superior de Arquitectura de Barcelona UPC (España 2010). Doctorando del Programa de Proyectos Avanzados de la ETSAM UPM (España, 2016 hasta la fecha). Ha dictado conferencias en el Graduate School of Design de la Universidad de Harvard (EEUU), Escola da Cidade (Brasil), la Facultad de Arquitectura de la Universidad de Oporto (Portugal) y el Recycl Art Center (Bélgica), entre otros. Premio Bienal Iberoamericana de Arquitectura y Urbanismo (IX BIAU) al mejor libro de arquitectura y el Architectural Book Awards organizado por el Deutsches Architekturmuseum de Frankfurt, ambos premios obtenidos el año 2014. Jurado de la X Bienal Iberoamericana de Arquitectura y Urbanismo 2016 realizada Sao Paulo, Brasil. Actualmente es profesor a jornada completa de la Escuela de Arquitectura de la Universidad de Talca. 
MÁRGENES DE ACCIÓN: EL PROCESO ARTESANAL COMO MÉTODO DE PROYECTO EN LA ARQUITECTURA CONTEMPORÁNEA DE PARAGUAY LIMITS OF ACTION: THE ARTISAN PROCESS AS PROJECT METHOD IN THE CONTEMPORARY ARCHITECTURE OF PARAGUAY José Luis Uribe Ortiz

\section{p.17 ON ISOLATION AS A CONDITION}

Paraguay is a land-locked country situated in the middle of the South American continent, surrounded by the respective cultures of three different countries: Bolivia to the north, Brazil to the east, and Argentina to the southeast, south and southwest. This particular location gives its culture an introspective character and an autonomy that seeks to distance itself from the countries that make up its borders. This encapsulation has manifested itself in different episodes of its history, such as the socialist insula of Doctor Francia during his time of national governance, the warlike attitude that has led them to fight against neighboring countries ${ }^{1}$ or the political enclosure of Stroessner during the most extensive dictatorship of Latin America².

In demographic terms, Paraguay has one of the sparsest populations compared to its Latin American peers, irregularly distributed throughout its territory, but mainly concentrated in the eastern region of Paraguay, proof of the inhospitable condition of the western region which is characterized by its warm temperature and dense jungle. According to Britez and Numan, in economic terms, Paraguay has the lowest gross domestic product in Latin America. Transparency International considers it the most corrupt country in the continent, and the second most-corrupt in the world. It is one of the poorest countries in Latin America ${ }^{3}$.

Considering this background, at a Latin American scale, Paraguay shows limited development when compared to other Latin American countries, in geographical, political, demographic and economic aspects. The isolation and geographic confinement that plagues the country has repercussions, resulting in a constant exclusion of Paraguay.

At a national level, Paraguay's internal structure exhibits fragmentation and polarization across the different disciplines of social sciences. Inequality has affected the inhabitants of Paraguay during the last few decades. It manifests itself through corruption, scarcity and poverty.

p.18 Despite Paraguay's significant social challenges, during the last few decades, they've achieved a degree of cultural development based on their own culture, unique from external references, a condition that has influenced a particular way of doing architecture.

\section{ON MATERIAL CULTURE AND ARTISAN PROCESSES}

The isolated condition that Paraguay manifests in addition to its scarcity of resources, is contracted for its generous material culture, which has been highlighted by various authors ${ }^{4}$. For the present study, it is pertinent to approach the concept of material imagination, illustrated by Gastón Bachelard in his book "Water and Dreams an Essay on the Imagination of Matter" ${ }^{\prime \prime}$, since it allows us to infer the opening that is provided by matter in a creative process. In architecture, matter can inform the project, as well as define the architectural expression of the work. The information that matter gives the project process can be established in two lines: on the one hand, through the material exploration, guided by the architect, and on the other hand, through intuition, dexterity and proximity to the material given by an artisan (figure 1).

We will consider the role of the artisan in the contemporary architectural narrative of Paraguay, and that is what will define the guiding thread of the present study. We will take distance from the image of the architects who have received an artisanal training ${ }^{6}$ and who incorporated that knowledge into their design processes, since this interpretation has blurred the authorship between architect and craftsman. We will add the points raised by Juhani Pallasmaa regarding the fact that in a creative work, both the artist and the artisan are directly involved with their bodies and existential

p.19 experiences, rather than focusing on an external and objectified problem ${ }^{7}$. This is how the figure of the craftsman emerges from anonymity and is enhanced in a synergy with the architect.

To narrow down the study, it is necessary to take a position on the artisanal term. According to the RAE, Real Academia Española, the Spanish term artesanal corresponds to products that are elaborated through traditional or manual techniques, without the intervention of an industrial process. According to this definition, we will keep distance from the notion of product that arises from it, and we will focus on craftsmanship as an experience, according to the point of view established by Richard Sennett when referring to craftsmanship as a process characterized by constant practice, where the head and body unite in a common mission linked to matter ${ }^{8}$. This idea reflects the logic of thinking with hands and an intellectual learning through the reiteration of an action of the body on the subject, a practice trained over time ${ }^{9}$. This position can be associated with the particular way of doing architecture in Paraguay, where throughout its history, it has been characterized by the search for a local architectural language based on the use of materials that the environment provided.

According to Gutiérrez, during Paraguayan architecture in the period of Hispanic domination, three major sectors assume the task of creating architecture: masters of works, architects of religious orders and architects of military engineers. Focusing on the masters of works, Gutierrez highlights the fact that "they are specially trained through the process of apprentice-official-teacher of the Spanish unions, who in Paraguay found possibilities to develop their skills with new materials and constructive systems" ${ }^{\prime 10}$. This apprentice-official-teacher relationship is based on the idea of 
craftsmanship proposed by Sennett, where the purpose is to establish an intellectual learning based on practice trained in time and constant repetitions, to which is added a great capacity of Paraguayan artisans to adapt to the economic, technological and material conditions of the time, new materials and construction systems ${ }^{11}$ (figure 2).

The artisan processes that are part of the architectural expression in Paraguay begin to obey the material logic provided by the immediate environment of the place, the incidence of artisanal knowledge as part of a synergy based on the exchange of experiences and experimentation developed among the artisan builder and the architect. Thus, a greater relationship of the work with the location and the human reality carried out by that architecture is defined, distancing itself from the logic of an architecture based only on the material collection that characterized its beginnings. For the publisher Florencia Rodríguez, the contemporary manifestation of contemporary architecture in Paraguay "is not an architecture of drawn detail, but claims to get dirty feet in the mud and look for a high degree of sophistication of material resources"12. This is how this way of projecting is, based on material manipulation where in a washed out, crude, and remote place, far away from the computers, it is possible to approach the plastic expressions that characterize contemporary architecture in Paraguay (figure 3).

\section{CONTEMPORARY ARCHITECTURE IN PARAGUAY}

Consulting the books on Latin American architectural history and theory or the exhibitions and biennials associated with the discipline, the architecture of Paraguay has always been relegated or simply absent, insisting on this isolated condition mentioned at the beginning of the study. It was not valued within the architectural production of the Latin American context and it did not recognize its own way of doing architecture. At the end of the nineties, after the political dictatorship of Stroessner and under a string of corrupt transitional governments, a particular way of doing architecture emerged, that distanced itself from the production developed in Latin America. From the hand of architects Javier Corvalán and Solano Benítez, emerges a series of small architectural works that provide their own language based on the original material culture of Paraguay, the recognition of new ways of interpreting ordinary materials and applying them in works granting a spatial dignity, plastic and material processes in which the architect developed a collaborative work with the craftsmen's own experience.

This has generated a critical mass that has raised international interest in the contemporary architectural scene in Paraguay ${ }^{13}$, that is part of a slow process of transmission of knowledge that had to go through various stages to later position itself not only nationally, but also internationally. This is how this architectural manifestation situated in the heart of South America contains characteristics of what Frampton deemed Critical Regionalism ${ }^{14}$ and that Zaera Polo groups under the concept of Material Fundamentalists in the graph of the article Ya Bien Entrado el Siglo XXI ¿Las Arquitecturas del Post-Capitalismo ${ }^{15}$. In this sense, this article does not intend to enhance the social architecture or recycling architecture label that during the last decades has received the work of some of the firms. On the other hand, the architectural analysis will be structured as a story that seeks to approach the complexity of the project processes studied and the impact on the expression of its architecture, of the artisan linked to the work, of a fundamental weight and role.

\section{Study of cases}

The present study of cases is framed in the work of three reports of Paraguayan offices of which certain partialities are of interest. The cases reviewed have, as a common element, the design of the architectural object in contexts of material abundance and economic scarcity, in addition to the incorporation of artisans who have direct participation in the architectural project. This craft participation is usually established along with the architect, in a dialogue based on exchange of experiences around the manual procedures involved in architecture, those that come from the hands of potters, masons, stonemasons and carpenters, who develop their work from the matter that surrounds them. Next, we will study the unique architectural elements of each work, broken down by three characteristic materials in that country, guiding the study from the incidence of the craft process to the architectural method.

On bricks as material

We will start the study by considering the brick as material and we will focus on the parasol of the Medical and Housing Clinic designed by the Elgué Study. It presents a ceramic fabric on the west façade supported by a single equilibrium point, linking the spatial situation of the street, the new construction and the pre-existing one. This architectural element defines a permeable screen whose function aims, on the one hand, at taking care of the excess light and heat coming from the west and, on the other hand, at configuring an element that defines the continuity of the facade of the whole, which also narrows the relationship between the public space and the private space (figure 4).

This mono-material screen is structured from a rectangular geometry that overlaps the building, defining a translucent façade that is made from a heavy element such as brick $^{16}$, whose material language is manifested by two 
types of grain: first, corresponding to an airtight brick wall resting on a spindle, suspending the weight of the entire façade in the air. The second grain of the brick is recognized on that closed geometry, which is adhered by a grid of vertical and zigzagging brick panels, leaving a space for ventilation between each brick. The ceramic membrane is suspended and does not touch the walls of the preexisting building or the natural floor where the architectural object is located. The access to the building is like a vacuum that allows the dialogue of the architecture with the scale of the inhabitant and consists of a rectangular cutout at the lower left end of the larger geometry (figure 5).

It should be noted that the plastic expression of the building is defined by a process of trial and error developed in the field, addressed from a series of material tests and subsequent project decisions in which both architect and craftsman participated, granting those discarded bricks a greater dignity, approaching the idea of defect as architectural expression. Another remarkable point is the geometry of the architectural element that is associated with the geometry of the indigenous basket weavings of Paraguay, characterized by its construction based on fabric and framework (figure 6).

\section{On wood as stacking material}

The study of the second case considers wood as matter applied to a structural architectural element such as the pillar, developed from a local material language associated with artisanal tasks. We will focus on the pillar of the studio house Las Mercedes by Lukas Fuster, who projects a particular architectural element as a result of an intervention that takes advantage of the subtraction of material developed on an old house located in the old town of Asunción, where the recycled material is incorporated back into the home from a series of domestic interventions, which shows the logic that matter is not created nor destroyed, but only transformed ${ }^{17}$. The only pillar incorporated in the house is presented under a constructive system that stacks and threads pieces of discarded wood by compression, using a pre-stressed steel rod giving shape to the shaft of the pillar. Both the base and the capital are defined by two concrete specimens located at the spot in which the pillar touches the floor and ceiling of the house, giving the pillar a dignity typical of a column that supports part of the house, just like the wall does as structural element present in rural Guarani housing (figure 7).

Materially, this part is composed of a sum of pieces corresponding to the smallest remains of the wooden braces,

p.23 that have been reused and that are concentrated in a single architectural element based on the architect's centrifugal action, similar to the approach proposed by Ramón Gutiérrez regarding the value, score and accumulations of material elements present in the Paraguayan Indian culture. The material composition of the column reconsiders the plastic language of the column, which usually has a continuous mono-material composition. In this case, it is constituted through superposition of pieces, defining an element that mediates between the slab and the ceiling, similar to the material stacking of the apacheta, accumulation of stones of Indians and mestizos of certain Andean regions. Finally, the architectural element developed by Fuster is a pillar based on the action of stacking, a game of words that insists on the accumulation and superposition that defines the architectural attitude (figure 8).

El Pilar of the Las Mercedes studio house is part of an architectural work of procedural nature, built over time, that takes into account the constant incidence of climate on the site, as well as the attentive look of its inhabitant to the daily environment and the functional elements that make up its architecture. The house is understood as a laboratory in which each architectural intervention attends a series of material and structural tests that affect their domestic habitation. In this case, the figure of the architect and inhabitant of the work is transformed into a sort of Animal laborans, in the words of Sennett, in whose domestic environment it is possible to see various manual works that give an account of Fuster's affinity for crafts, where his house study is a constant test of methods of building (figure 9).

\section{On earth as compaction material}

To close the study of cases, we will refer to earth as project material of the exterior wall of the Takurú House of José Cubilla, which corresponds to an architectural element formed by the compaction of earth, and which builds the perimeter that defines the wholeness of the House. This element is based on a local construction system like the rammed earth, an old technique that consists of constructing load-bearing walls from moist clayish earth compacted by means of a rammer, which defines its base thanks to a wooden formwork. The excavation of a large pit provides the raw material to build the extension of the wall, and the ground becomes a paramento. Matter acquires geometry in order to trace the house from a wall that provides continuity and rhythm, to a spatial sequence that rests in its central void (figure 10).

The exterior wall, as part of the wall box of this work, defines a hermetic condition of the daily life of the interior of the house, in contrast with the opening of the extension of the natural landscape where it is located. This idea of a perimeter element formed from a compacted material, makes it possible to establish a relationship with Paraguayan

p.24 indigenous pottery, in particular with vessels, both in the conception of a central void that constitutes the rampart wall and as architectural definition, as well as in the interior vacuum present in the composition of indigenous pottery vessels. In both cases, an intention to define a container element and a contained space, is observed, in which an air is sought, a possible interior, as in the case of the Takurú house, where a small interior patio is equated with the case of the vessel and the empty space inside a material container (figure 11).

Another relationship between the mud wall and the indigenous pottery produced in that territory has to do with the material condition of both cases, where the reddish terracotta and the grooves of the joints of the walls are similar to the incisions proper to the decoration of the Guarani pottery. From the tapial as construction system, it is possible to observe the material strata in the wall, its granulometry and a suggestive reddish color, elements that mediate 
between the architecture and the kindness of the earth where the work is located. During the construction process of this architectural element, it is possible to observe the contemporary development of an ancient artisanal construction practice where body, material, place and architecture converge. In the process of manufacturing the indigenous vessels, a sculptural condition, typical of handwork, appears. The vessel is a container that contains air and the walls of the Takurú House trace a container that also contains air, but it is an air that expects to be inhabited.

Materially, it is an architectural element constituted of weight, appropriating the constructive logic based on the stereotomy of Semper's bases, by means of a mono-material element. A wall made from the constant knocking in the process of work in the field, a manual process developed between the architect and the craftsman, where the primitive material expression of the work resonates, which manifests itself crudely and grossly towards the elements (figure 12).

This architectural element corroborates the projective attitude of contemporary architects in Paraguay to build with the elements gathered from around, by Gutierrez, and the logic of the centripetal from the definition of a concentrated and dense architectural element, constituted by the material wealth of the surroundings.

\section{SOME NOTES AS CONCLUSION}

On the incidence of the body in architectural expression

After this review of cases, it is necessary to highlight the importance that Peter Zumthor gives to the body in architecture. The sensory effect that the material detonates in the architect allows to "gather things and materials from the world so that, together, they create this space. For me, it is like anatomy. Actually, when talking about the body, I do it in the literal sense of the word"18. Zumthor's definitions incorporate a material component and a participation of the body in the formal definition, suggesting a manual interaction in the constructive process. It is like, on the one hand, the material component delivers an information that is decoded by the body of the inhabitant when crossing the work ${ }^{19}$, which Pallasmaa recognizes as sensory experiences, becomes integrated through the body ${ }^{20}$. On the other hand, Alvaro Aalto gives importance to the human as part of the architectural process, where "the human factor has always been part of the architecture and that, in a deeper sense, it has even been indispensable, because without its contribution, one could not express the richness of life and its positive qualities"21.

According to what Zumthor, Pallasmaa and Aalto raise, we could conclude that the craftsman, from his corporality in contact with the material, contributes to the architectural expression of the constructed work, which in the case of contemporary architecture in Paraguay, has a direct impact on his architectural language, which is gestated from material experimentation in the field, as described by Rodríguez: "This is not an architecture of drawn detail, but claims to get dirty feet in the mud and look for a high degree of sophistication of material resources"22. It is the body that defines the habitable scale of the spaces during the design stage, the plastic expression of the building in its construction process, and the material definition of the architectural object once it has been constructed.

\section{On hapticity in architecture}

Finally, the participation of the artisan within the materialization of the project provides a tactile quality to the experience of tracing the spatial sequence of an architecture. For Le Corbusier, stone, brick, wood or iron have different potentials, sentimental and physical energies, where the permanent contact of the inhabitant with matter is a fundamental need ${ }^{23}$. Pallasmaa recognizes two variables that articulate the sensitive interaction of the inhabitant with the architectural object: first, the touch as sensory element that integrates our experience of the world with our interior. Second, the surfaces of natural materials that allow us to convince ourselves of the veracity of the material, in an era characterized by the smoothness of the standard construction. The surface of the material defines the various spaces of the architectural object, and guides a spatial sequence based on the experience of living in material contact with the work. Deplazes gives value to the architect Mandfred Sack's description of the haptic dimension of architecture: "Again and again, the sensuality of the material, of matter, becomes present: how it feels to the touch, to the eye. If it is matt or bright, if it smells, if it is hard, soft, elastic, cold or hot, smooth or rough, what are its colors, what structures are exhibited on its surface"24.

The above can be understood as the sincerity of the material surface, which is always shown in a direct, raw and visceral way to the inhabitant, whose tactile definition comes as a consequence of a craft work in the process of construction of architectural work, like the architect and Paraguayan artist Carlos Colombino works the surface of his matrices to develop his woodcuts, whose textures "arise at each stroke of the blade. The planes multiply. The relief is enriched, and the color concurs to suggest lights and shadows"25. A similar way of operating is developed by contemporary Paraguayan architects, who recognize in vernacular construction processes a series of values, as shown in this study's analyzed works, where walls, floors and skies are configured from repetitive elements like pieces of brick, wood or pieces made of compressed earth, providing roughness and grooves in the expression of the surface of the architecture, that Rodríguez calls "a kind of monochrome patchwork that is perceived as tied but that contains a catalog of different textures. This creates undeniably vernacular atmospheres and experiences" 26 . A unique living experience, guided by a great material load that has been shaped with great skill by each craftsman whose trade has been built over time, just like the identity of contemporary architecture in Paraguay has been built.

On the centripetal logic of building with the matter that is around

The architectural elements examined account for different material and constructive actions developed from the collaboration between the architect and the artisans. We can mention the action of entramar in the parasol of the 
Medical Clinic and Housing by Estudio Elgué, the stack that defines the pillar of the Casa Estudio Las Mercedes by Lukas Fuster and the densification that can be recognized in the muraria expression of Casa Takurú. Each of these material actions has been developed manually, forming a constructive centripetal logic that tends to concentrate direct and indirect references of the local culture of Paraguay in these architectural elements. A different approach to the condition of isolation that Paraguay presents as a culture that tends to centrifuge external cultural references.

1. I am referring to the war against the Triple Alliance (1864 - 1870) and the Chaco War (1932 - 1935).

2. The Stroessner dictatorship was developed between 1954 and 1989, with an extension of 35 years.

3. BRITEZ, Edwin. NUMAN, Javier. El Paraguay actual. 1998- 2010. Asunción: El Lector. 2010.

4. Juan Giuria puts forth his woods, stones and brick of Paraguay, proposing that: "It is estimated that more than seventy percent of its surface is covered by forests of magnificent woods, suitable for all kinds of works. As for materials of stone origin, it is indisputable that there are quarries of excellent sandstone, as proven by certain works carried out by the Jesuits, in which enormous walls can still be seen executed with blocks of that stone. During the Hispanic era, and even in later years, there was a great use of clay, either in the form of adobe or simply as rammed earth. The use of brick was developed from the independence onward". For researcher Ramón Gutierrez, the geographical context influenced a material culture based on the groping, accumulation and hoarding of materials, highlighting that: "The materials available from the beginning in the Paraguayan territory, were those of collection: wood, cane, straw, mud and stone".

5. BACHELARD, Gastón. El agua y los sueños. Ensayo sobre la imaginación de la materia. Madrid: Fondo de Cultura Económica de España Editorial. 2005.

6. I am referring to architects like Carlo Scarpa, who was trained for twenty years with the artisan training of the master glassmakers of Murano, or the case of Peter Zumthor, who claims his origins as the son of a craftsman (cabinetmaker in Basel), who was trained as a carpenter.

7. PALLASMAA, Juhani. Los ojos de la piel: La arquitectura y los sentidos. Barcelona: Gustavo Gili Editorial, 2012.

8. This idea reflects the union of the hand and the head applied on the subject, which allows a link between manual practice and ability to innovate and solve problems nowadays from a material logic.

9. SENNETT, Richard. El artesano. Barcelona: Editorial Anagrama. 2009.

10. GUTIÉRREZ, Ramón. Historia de la arquitectura del Paraguay 1537-1911. Asunción: Municipalidad de Asunción Editorial. 2010, p.51.

11. Cecilia Román and Andrés Careaga affirm that: "Paraguayan architects who have contributed to the discipline first had to devise ways to teach their masons new ways of working. (...) A re-elaboration process begins, understanding the traditional technique first, mastering it and then transforming it, and above all, being able to explain it and put it into practice satisfactorily".

12. RODRÍGUEZ, Florencia. Nuevas prácticas en Asunción. In: PLOT. Buenos Aires: Piedra, Papel y Tijera SA, number 26, 2015, p. 28.

13. Ibidem, p. 26. According to Rodríguez, there is an architectural openness in Paraguay: "It has a correlation with certain exchanges that were generated over time through workshops, conferences, publications and conversations. We could highlight some facts such as the selection of finalists that took place on the occasion of the second Mies van der Rohe Award for Latin America. Angelo Bucci from Brazil, Rafael Iglesia from Argentina, Alejandro Aravena from Chile and Solano Benítez from Paraguay established themselves, from that moment on, as a representative group of some of the transformations and proposals of the region. This point opened the dialogue between people and between the body of works that gained visibility and new layers of meaning when their putting into action was activated".

14. As a reaction to the international style, Frampton postulates critical regionalism approaching an architecture that counteracts the lack of place and lack of identity in modern architecture, by using the geographical context and material of the building as project starting point, taking up the work of the artisan who, from his manual processes, defines the particularities of an architecture that struggles with the tendency to standardize.

15. ZAERA POLO, Alejandro. Ya Bien Entrado el Siglo XXI ¿Las Arquitecturas del Post-Capitalismo? In: El Croquis. Madrid: El Croquis Editorial, number 187, 2016, p 252-287. 16. It must be taken into account that, in this work, the discard brick is used as a material match. The discard brick is one that, when it is positioned on the level of the natural floor of the drying yards, it shrinks due to heat, generating a deformation of the material unit. Being a defective brick, it has a lower cost, which helps promote an architecture based on the economy of resources.

17. DE LAVOISIER, Antonine-Laurent. Tratado de Química (Reissue). Valladolid: Maxtor Editorial. 2009.

18. ZUMTHOR, Peter. Atmosferas. Barcelona: Gustavo Gili Editorial. 2006, p. 22.

19. PALLASMAA, J. Op. cit., supra, note 7, p. 68. In the words of Pallasmaa "Skin reads the texture, weight, density and temperature of matter".

20. Ibídem, p. 50.

21. AALTO, Alvar. Alvar Aalto: De palabra y por escrito. Madrid: El Croquis Editorial. 2000.

22. RODRÍGUEZ, Florencia. Op. cit., supra, note 12, p. 28.

23. LE CORBUSIER. Cuando las catedrales eran blancas. Buenos Aires: Poseidón Editorial. 1963.

24. DEPLAZES. Andrea. Construir la arquitectura. Del material en bruto al edificio. Barcelona: Gustavo Gili Editorial. 2010, p. 19.

25. PLÁ, Josefina. Carlos Colombino, Paraguayan painter. En: Humboldt. Hamburgo: Goethe Institut Editorial, number 39, 1969. p. 97

26. RODRÍGUEZ, Florencia. Op. cit. supra, note 12, p. 28. 


\section{Autor imagen y fuente bibliográfica de procedencia}

Información facilitada por los autores de los artículos:

página 18, 1 (Crédito fotográfico gentileza de Federico Cairoli); página 19, 2 y página 20, 3 (Crédito fotográfico José Luis Uribe Ortiz); página 21, 4 (Crédito fotográfico gentileza de Berenice Gómez Crosa), 5 (Crédito boceto detalle constructivo gentileza de Luis Elgué); página 22, 6 (Crédito fotográfico gentileza de Estudio Elgué), 7 (Crédito boceto detalle constructivo gentileza de Lukas Fuster); página 23, 8 y 9 (Crédito fotográfico gentileza de Federico Cairoli), 10 (Crédito boceto detalle constructivo gentileza de José Cubilla); página 24, 11 y 12 (Crédito fotográfico gentileza de Federico Cairoli); página 29, 1 (Biblioteca Nacional de Australia. P490/7. 1918); página 30, 2, página 31, 3 y página 32, 4 (Javier Mosquera González); página 33, 5 (Biblioteca Nacional de Australia. PIC/9929/2029 LOC Cold Store PIC NICH), 6 (Biblioteca Nacional de Australia. PIC/9929/2110); página 34, 7 (Javier Mosquera González); página 35, 8 (Biblioteca Nacional de Australia. PIC/P2145); página 36, 9 (WikimediaCommons. Autor: Martyman); página 38, 10 (Javier Mosquera González); página 45, 1 (Elaboración propia); página 49, 2 (Elaboración propia, basado en información contenida en: MANNICHE, Peter, Living democracy in Denmark: independent farmers, farmer's cooperation, the folk high schools, cooperation in towns, social and cultural activities, social legislation, a Danish village. 2. ed. Copenhagen: G.E.C. Gad Pub., 1970. ISBN 0837139856), 3 (Elaboración propia, basado en la información contenida en: RUONAVAARA, Hannu. Home ownership and the Nordic housing policies in the 'Retrenchment phase'. En: Conference Building on Housing Ownership, Delft 2008; y en: TSENKOVA, Sasha; VESTERGAARD, Hedvig. Social Housing Provision in Copenhagen. Artículo presentado en ENHR 2011, Toulousse, France); página 50, 4 (Elaboración propia, a partir de la documentación original del proyecto aprobada por las autoridades de Copenhague, En base a la documentación cedida por Fællestegnestuen), 5 (Elaboración propia); página 51, 6 y 7 (Documentación cedida por Fællestegnestuen), 8 (HOLMBERG, Hartvig, ed. -indret selv Deres bolig. Copenhague: KAB, 1979, pp. 8-9); página 52, 9 (Documentación cedida por Fællestegnestuen), 10 (HOLMBERG, Hartvig, ed. -indret selv Deres bolig. Copenhague: KAB, 1979, portada y p. 43); página 53, 11 (HOLMBERG, Hartvig, ed. -indret selv Deres bolig. Copenhagen: KAB, 1979, p. 26), 12 (Elaboración propia); página 59, 1 (Parte superior: The American Woman's Home or Principles of Domestic Science Being a Guide to the Formation and Maintenance of Economical Healthful Beautiful and Christian Homes. New York: J. B. Ford and Company, 1869; The New Housekeeping: Efficiency Studies in Home Management. Garden City-New York: Doubleday, Page \& Company, 1913; The Psychology of Management: The Function of the Mind in Determining, Teaching and Installing Methods of Least Waste. New York: Sturgis \& Walton Company, 1914. Parte inferior: Catharine Beecher (Wikipedia Commons), Christine Frederick (Christine Frederick Archive, Schlesinger Library, Radcliffe Institute, Harvard University), Lillian Gilbreth (Lillian Moller Gilbreth Papers, Sophia Smith Collection, Northampton)); página 60, 2 y página 61, 3 (The American Woman's Home or Principles of Domestic Science Being a Guide to the Formation and Maintenance of Economical Healthful Beautiful and Christian Homes. New York: J. B. Ford and Company, 1869, p. 26, 37, 40); página 62, 4 (Christine Frederick Archive, Schlesinger Library, Radcliffe Institute, Harvard University); página 63, 5 (FREDERICK, Christine. The New Housekeeping: Efficiency Studies in Home Management. Garden City-New York: Doubleday, Page \& Company, 1913, p. 53); página 64, 6 (FREDERICK, Christine. Household Engineering: Scientific Management in the Home. Chicago: American School of Home Economics, 1920); página 65, 7 (Kheel Center for Labor-Management Documentation and Archives, Cornell University Library, Management Engineering), 8 (National Museum of American History, Behring Center, Division of Work and Industry Collection); página 66, 9 y 10 (Elaboración realizada por Carmen Espegel); página 67, 11 (Izquierda: BEECHER, Catharine y BEECHER STOWE, Harriet. The American Woman's Home or Principles of Domestic Science Being a Guide to the Formation and Maintenance of Economical Healthful Beautiful and Christian Homes. New York: J. B. Ford and Company, 1869, p. 23. Derecha: Siedlung Römerstad 1927-1928. Das Neue Frankfurt, n.4-5, Abril-Mayo, 1930, p. 76); página 68, 12 (Izquierda: HAYDEN, Dolores. The Grand Domestic Revolution: A History of Feminist Designs for American Homes, Neighborhoods, and Cities. Cambridge (Massachusetts): MIT Press, 1981, p. 30. Derecha: Ernst May und das Neue Frankfurt, 1925-1930. Catálogo de la exposición en el Deutsches Architekturmuseum Frankfurt am Main. Berlin: Wilhelm Ernst \& Sohn Verlag, 1986, p. 153); página 69, 13 (Superior izquierda y derecha: Restitución gráfica realizada por Carmen Espegel. Inferior izquierda: BEECHER, Catharine y BEECHER STOWE, Harriet. The American Woman's Home or Principles of Domestic Science Being a Guide to the Formation and Maintenance of Economical Healthful Beautiful and Christian Homes. New York: J. B. Ford and Company, 1869, pp. 28-30. Inferior derecha: Das Neue Frankfurt, n.6, Junio, 1929, p. 128), 14 (Izquierda: FREDERICK, Christine. Household Engineering: Scientific Management in the Home. Chicago: American School of Home Economics, 1920, p. 32. Derecha: Margarete SchütteLihotzky: Frankfurter Küche, 1926. Colección y Archivo de Margarete Schütte-Lihotzky en la Universidad de Artes Aplicadas de Viena); página 70, 15 (Izquierda: Original films of Frank and Lillian Gilbreth, promovida por Chicago Chapter, Society for the Advancement of Management, 1910-1924. San Francisco: National Film Preservation Foundation. Derecha: Neues Bauen in Frankfurt am Main, dirigida por Paul Wolff. Frankfurt: Wolff-Film, 1928); página 75, 1 (Enrique Jesús Fernández-Vivancos González a partir del plano de Tapiola de Aarne Ervi de 1963); página 76, 2 (Le Carré Bleu. 1960 nº 3); página 77, 3 y 4 (Museum of Finnish Architecture [MFA]); página 78, 5 (Le Carré Bleu.1958 nº 1 pp. 2-3. Fotógrafo: PIETINEN, Otson); página 79, 6 y 7, página 80, 8, 9 y 10 y página 81, 11 y 12 (Museum of Finnish Architecture [MFA]); página 82, 13 (Museum of Finnish Architecture [MFA]. Fotógrafo: LEHTONEN, Kai R); página 83, 14 (Museum of Finnish Architecture [MFA]); página 84, 15 (PIETILÄ, Reima. Noción Imagen Idea. Espoo: Teknillisen Korkeakoulun Ylioppilaskunta. 1975. Lección 5 y Lección 16); página 90, 1 (Natalia Matesanz Ventura); página 93, 2 y 3 (Fotograma del documental dirigido por Christina Holmes. 2015 [en línea] https://www.youtube.com/watch?v=bB-3Bp2pWh8); página 103, 1 (https://lebbeuswoods.files.wordpress.com/2011/06/Iw-mugshot1.jpg [en línea] [consulta: 22-08-2017]. Disponible en: https://lebbeuswoods.wordpress.com/2011/06/29/anti-journey-to-architecture-1/), 2 (https://lebbeuswoods.wordpress. com/2009/10/06/notebook-01-3-the-last/); página 106, 3 (https://lebbeuswoods.files.wordpress.com/2012/01/lbw-411.jpg[en línea] [consulta: 22-08-2017]. Disponible en: https://lebbeuswoods.wordpress.com/2012/01/02/origins/); página 108, 4 (https://lebbeuswoods.wordpress.com/2009/09/27/the-vagrant-light-of-stars/ [consulta: 22-08-2017]), 5 (https://lebbeuswoods.files.wordpress.com/2010/11/dwg-1.jpg [en línea] [consulta: 22-08-2017]. Disponible en: https://lebbeuswoods.wordpress. com/2010/11/02/drawings-stories/); página 109, 6 (https://lebbeuswoods.wordpress.com/2009/08/01/meta-institutes/ [consulta: 22-08-2017]); página 110, 7 (https:// lebbeuswoods.wordpress.com/2009/03/15/notebook-97-3/ [consulta: 22-08-2017]), 8 (https://lebbeuswoods.wordpress.com/2009/12/19/storm-watch/ [consulta: 2208-2017]); página 111, 9 (https://lebbeuswoods.wordpress.com/2009/06/05/architecture-of-energy/ [consulta: 22-08-2017]), 10 (https://lebbeuswoods.wordpress. com/2011/02/15/a-space-of-light-2/ [consulta: 22-08-2017]); página 112, 11 (https://lebbeuswoods.files.wordpress.com/2012/03/Itpav-may-30-3.jpg [en línea] [consulta: 22-08-2017]. Disponible en: https://lebbeuswoods.wordpress.com/2012/03/25/light-pavilion-under-construction/); página 113, 12 (https://lebbeuswoods.files.wordpress. com/2011/01/cdusk1day.jpg [en línea] [consulta: 22-08-2017] Disponible en: https://lebbeuswoods.wordpress.com/2011/02/15/a-space-of-light-2/); página 114, 13 (https://lebbeuswoods.wordpress.com/2009/09/19/line-up/ [consulta: 22-08-2017]), 14 (https://lebbeuswoods.files.wordpress.com/2011/02/int-5-11.jpg [en línea] [consulta: 22-08-2017]. Disponible en: https://lebbeuswoods.wordpress.com/2011/02/15/a-space-of-light-2/), 15 (Fotografía: Shu He. Imagen cedida por Steven Holl Architects) 